Supplement of Atmos. Chem. Phys., 21, 9329-9342, 2021

https://doi.org/10.5194/acp-21-9329-2021-supplement

(C) Author(s) 2021. CC BY 4.0 License.

(c) (1)

Supplement of

\title{
Technical note: Sea salt interference with black carbon quantification in snow samples using the single particle soot photometer
}

Marco Zanatta et al.

Correspondence to: Andreas Herber (andreas.herber@awi.de)

The copyright of individual parts of the supplement might differ from the article licence. 


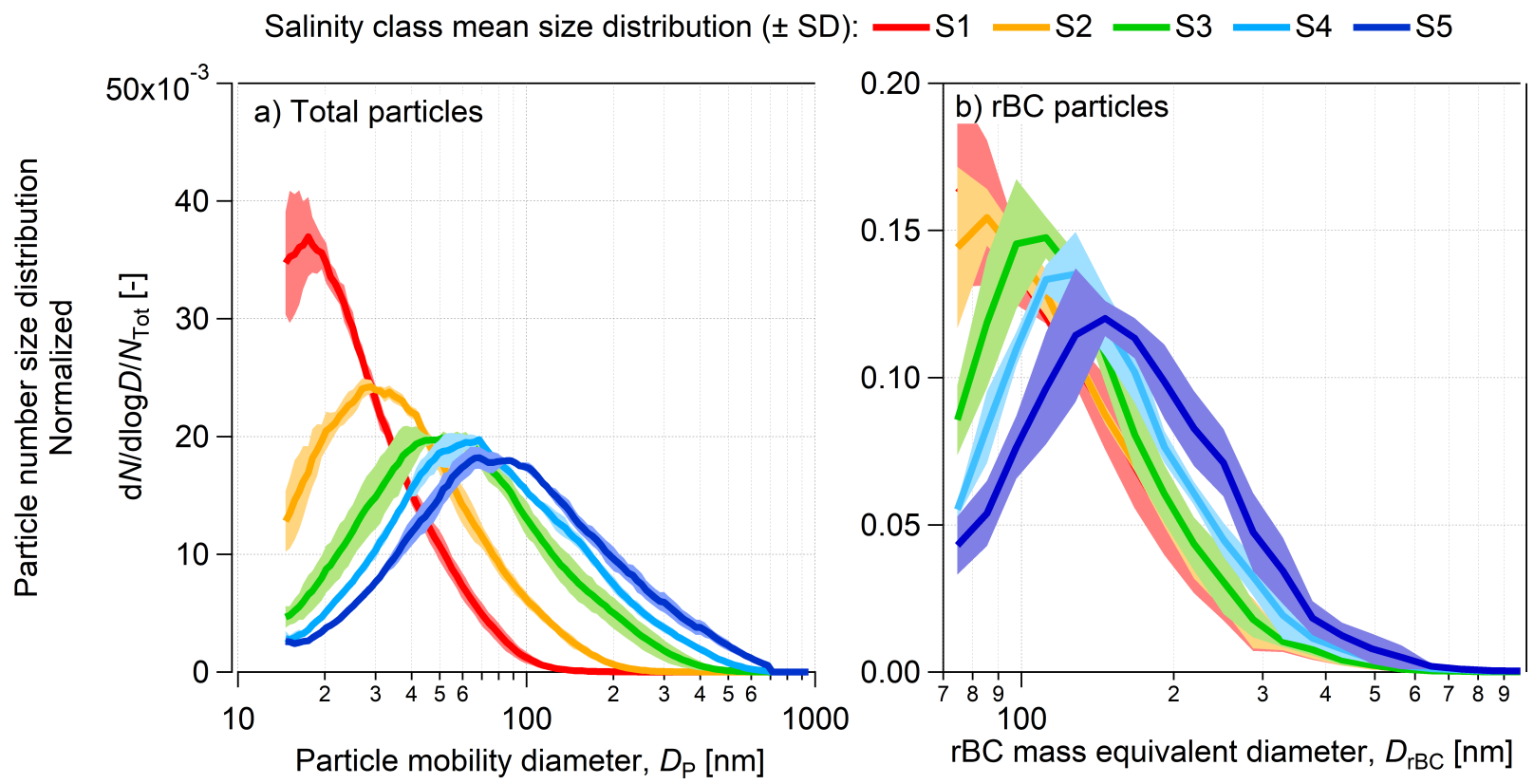

Figure S1 Normalized size distribution of total (a) and rBC particles (b) aerosolized from PASCAL snow samples grouped in salinity classes. Total particles measured with the SPMS in the $14-680 \mathrm{~nm}$ diameter range $\mathrm{nm}$. $\mathrm{rBC}$ particles measure with the SP2 in the $5 \quad 70-1000 \mathrm{~nm}$ diameter range. 


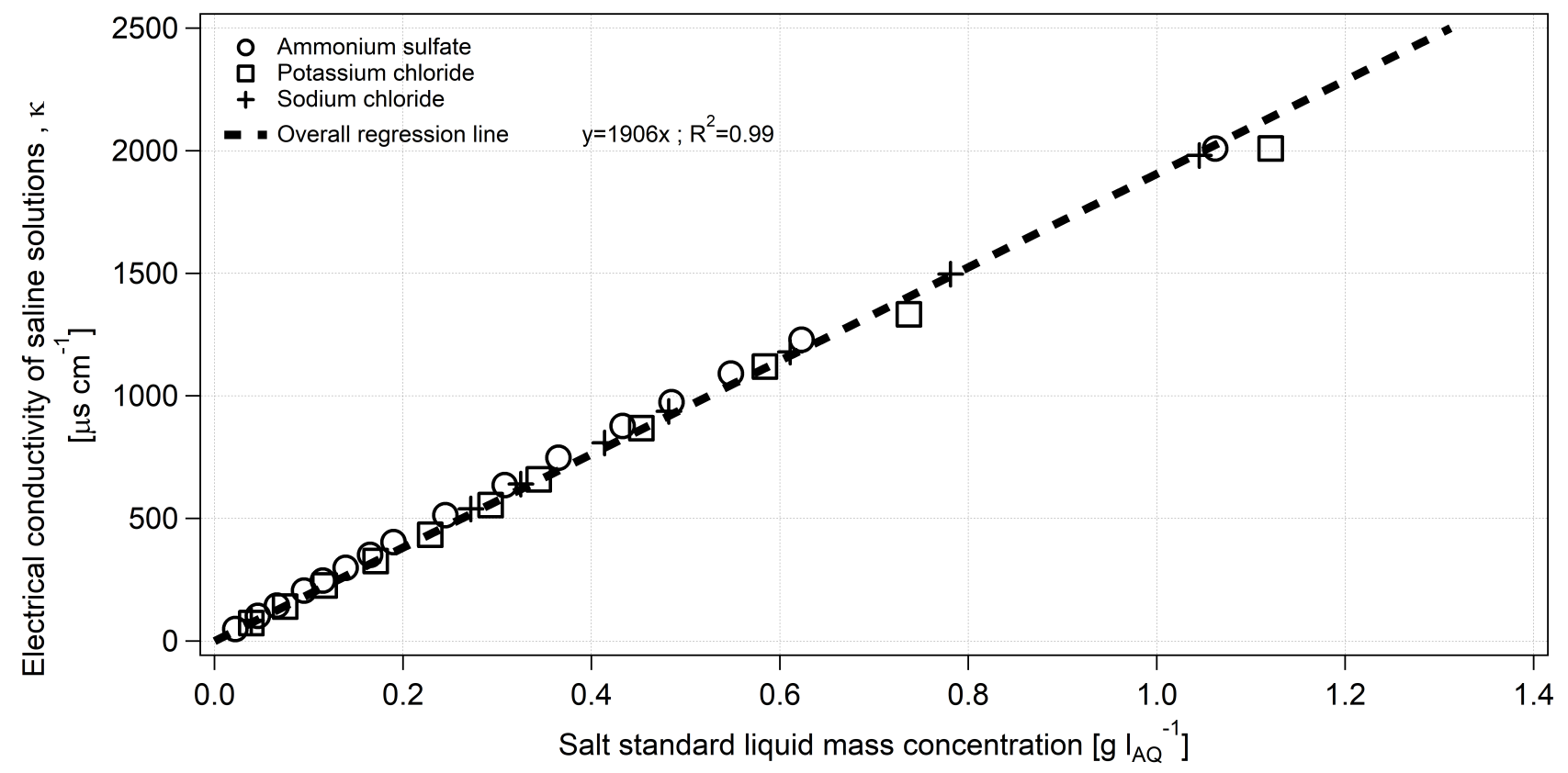

Figure S2 Electrical conductivity of saline solutions as a function of salt liquid mass concentration. Electrical conductivity measured 10 between 22 and $23^{\circ} \mathrm{C}$. 


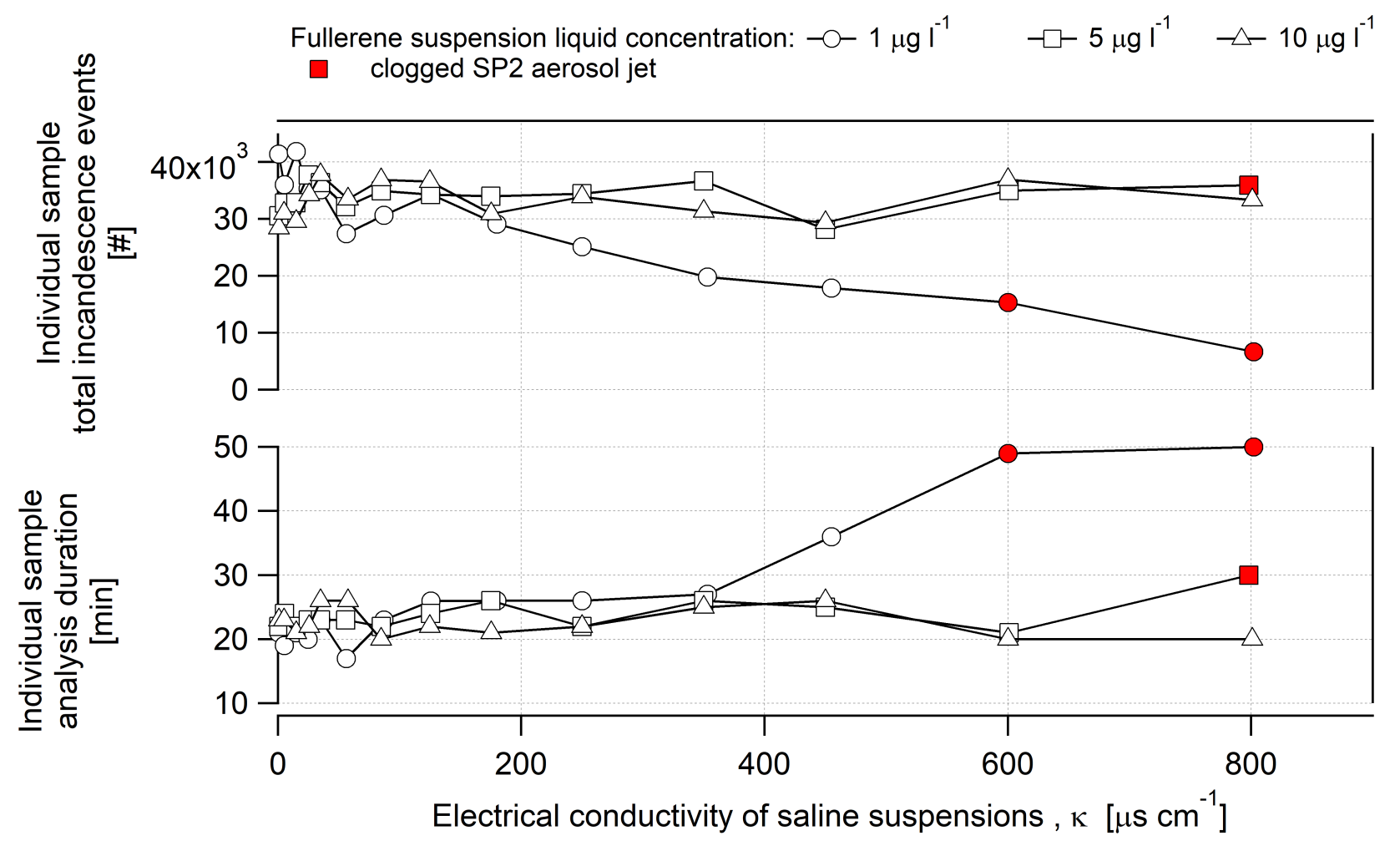

Figure S3 Total incandescence events and analysis duration of fullerene soot suspensions at different concentration and in increasing 15 electrical conductivity. 


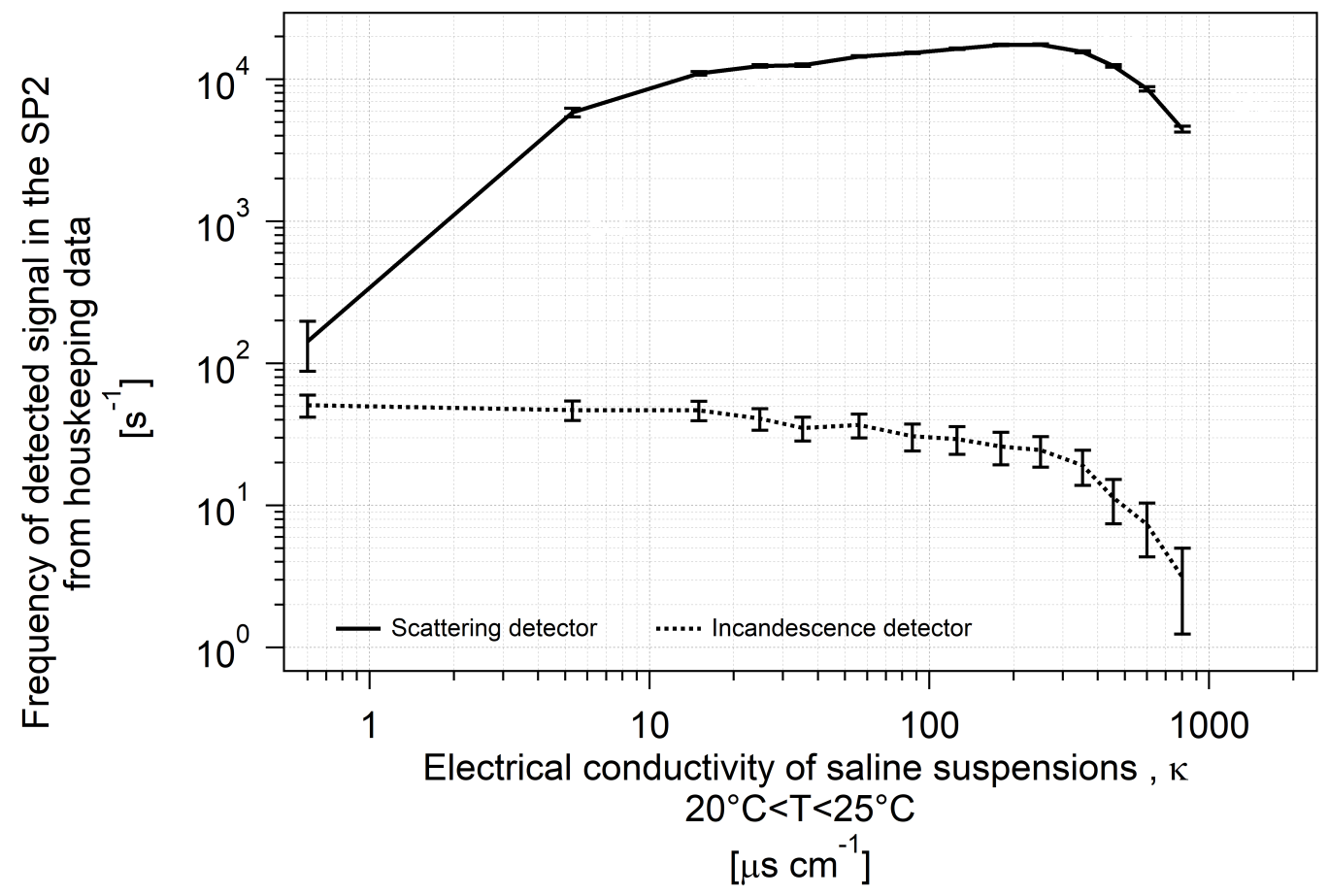

Figure S4 Frequency of detected scattering and incandescence signal in the SP2 recorded in the housekeeping data during the analysis of fullerene soot suspension $\left(10 \mu \mathrm{g} \mathrm{l}^{-1}\right)$ with increasing $\mathrm{NaCl}$ concentration. Signal acquisition triggered over the scattering detector. 
Electrical conductivity of fullerene suspension $, \kappa\left[\mu \mathrm{sm}^{-1}\right]:=0-50-100-200-400-600-800$ Fullerene suspension mass concentration: $10 \mu \mathrm{gl}^{-1}$

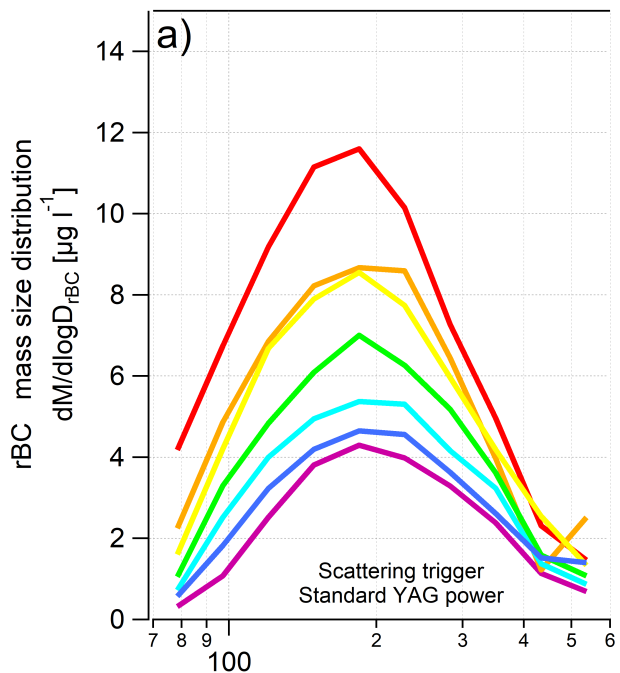

25

Figure S5 rBC mass size distribution for different SP2 settings. a) acquisition triggered on the scattering detector with standard

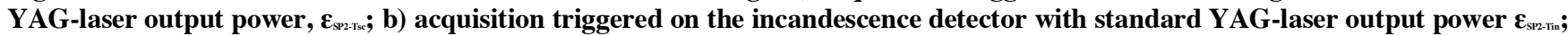

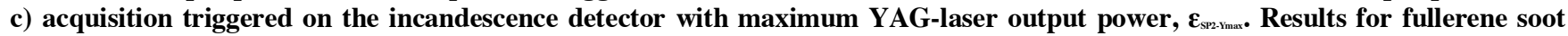
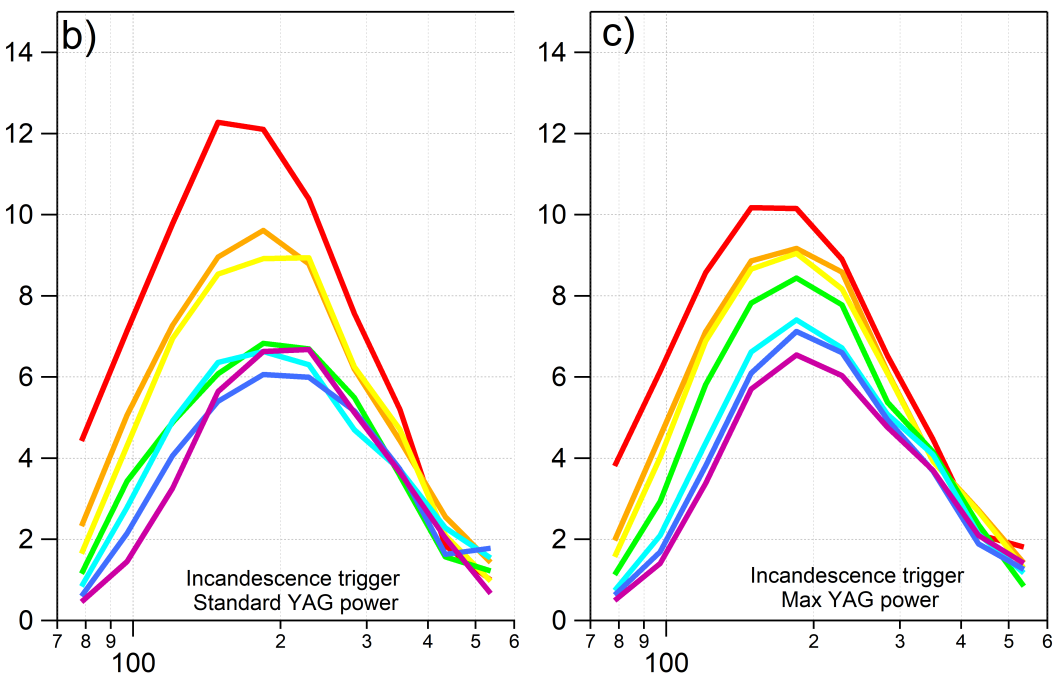

Black carbon mass equivalent diameter, $D_{\mathrm{rBC}}[\mathrm{nm}]$ suspensions with a concentration of $10 \mu \mathrm{gl}^{-1}$. 
Table S1 Theoretical coating thickness of BC particles suspended from saline suspensions at increasing electrical conductivity ( $x$ ) calculated assuming: concentric core-shell geometry, single BC particle-containing droplet, droplet diameter $\left(D_{\mathrm{v}}\right)$ of $5 \mu \mathrm{m}, 8 \mu \mathrm{m}$ and $12 \mu \mathrm{m}$.

\begin{tabular}{|c|c|c|c|c|c|c|c|c|c|c|c|c|c|c|c|}
\hline \multirow{3}{*}{$\begin{array}{l}x \\
{\left[\mu \mathrm{S} \mathrm{cm}^{-1}\right]}\end{array}$} & \multicolumn{15}{|c|}{ Coating thickness [nm] } \\
\hline & \multicolumn{3}{|c|}{$\begin{array}{l}D_{\text {rвс }} \\
100 \mathrm{~nm}\end{array}$} & \multicolumn{3}{|c|}{$\begin{array}{l}D_{\text {вс }} \\
200 \mathrm{~nm}\end{array}$} & \multicolumn{3}{|c|}{$\begin{array}{l}D_{\text {rвс }} \\
300 \mathrm{~nm} \\
\end{array}$} & \multicolumn{3}{|c|}{$\begin{array}{l}D_{\text {вас }} \\
400 \mathrm{~nm}\end{array}$} & \multicolumn{3}{|c|}{$\begin{array}{l}D_{\text {вас }} \\
500 \mathrm{~nm}\end{array}$} \\
\hline & $\begin{array}{l}\mathrm{D}_{\mathrm{D}} \\
5 \mu \mathrm{m}\end{array}$ & $\begin{array}{l}\mathrm{D}_{\mathrm{D}} \\
8 \mu \mathrm{m}\end{array}$ & $\begin{array}{l}\mathrm{D}_{\mathrm{D}} \\
12 \mu \mathrm{m}\end{array}$ & $\begin{array}{l}\mathrm{D}_{\mathrm{D}} \\
5 \mu \mathrm{m}\end{array}$ & $\begin{array}{l}\mathrm{D}_{\mathrm{D}} \\
8 \mu \mathrm{m}\end{array}$ & $\begin{array}{l}\mathrm{D}_{\mathrm{v}} \\
12 \mu \mathrm{m}\end{array}$ & $\begin{array}{l}\mathrm{D}_{\mathrm{o}} \\
5 \mu \mathrm{m}\end{array}$ & $\begin{array}{l}\mathrm{D}_{\mathrm{b}} \\
8 \mu \mathrm{m}\end{array}$ & $\begin{array}{l}\mathrm{D}_{\mathrm{v}} \\
12 \mu \mathrm{m}\end{array}$ & $\begin{array}{l}\mathrm{D}_{\mathrm{b}} \\
5 \mu \mathrm{m}\end{array}$ & $\begin{array}{l}\mathrm{D}_{\mathrm{b}} \\
8 \mu \mathrm{m}\end{array}$ & $\begin{array}{l}\mathrm{D}_{\mathrm{D}} \\
12 \mu \mathrm{m}\end{array}$ & $\begin{array}{l}\mathrm{D}_{\circ} \\
5 \mu \mathrm{m}\end{array}$ & $\begin{array}{l}\mathrm{D}_{\mathrm{b}} \\
8 \mu \mathrm{m}\end{array}$ & $\begin{array}{l}\mathrm{D}_{\mathrm{b}} \\
12 \mu \mathrm{m}\end{array}$ \\
\hline 0 & 0 & 0 & 0 & 0 & 0 & 0 & 0 & 0 & 0 & 0 & 0 & 0 & 0 & 0 & 0 \\
\hline 50 & 18 & 47 & 90 & 6 & 21 & 54 & 3 & 11 & 32 & 2 & 6 & 20 & 1 & 4 & 13 \\
\hline 100 & 30 & 69 & 125 & 11 & 37 & 84 & 5 & 20 & 55 & 3 & 12 & 37 & 2 & 8 & 25 \\
\hline 200 & 46 & 98 & 170 & 21 & 60 & 126 & 10 & 36 & 90 & 6 & 23 & 64 & 4 & 16 & 47 \\
\hline 400 & 68 & 135 & 226 & 36 & 93 & 180 & 20 & 62 & 140 & 12 & 42 & 107 & 8 & 30 & 82 \\
\hline 600 & 84 & 161 & 266 & 49 & 118 & 219 & 28 & 83 & 177 & 17 & 59 & 141 & 12 & 42 & 111 \\
\hline 800 & 97 & 182 & 298 & 59 & 138 & 250 & 36 & 101 & 207 & 23 & 73 & 168 & 15 & 54 & 136 \\
\hline
\end{tabular}


Table S2 Theoretical coating-rBC mass ratio of BC particles suspended from saline suspensions at increasing electrical conductivity $(\varkappa)$ calculated assuming: concentric core-shell geometry, single BC particle-containing droplet, droplet diameter $\left(D_{\mathrm{v}}\right)$ of $5 \mu \mathrm{m}, 8 \mu \mathrm{m}$ and $12 \mu \mathrm{m}$.

\begin{tabular}{|c|c|c|c|c|c|c|c|c|c|c|c|c|c|c|c|}
\hline \multirow{3}{*}{$\begin{array}{l}x \\
{\left[\mu \mathrm{S} \mathrm{cm}^{-1}\right]}\end{array}$} & \multicolumn{15}{|c|}{ Coating-rBC mass ratio $[\mathrm{nm}]$} \\
\hline & \multicolumn{3}{|c|}{$\begin{array}{l}D_{\text {rвс }} \\
100 \mathrm{~nm}\end{array}$} & \multicolumn{3}{|c|}{$\begin{array}{l}D_{\text {вс }} \\
200 \mathrm{~nm}\end{array}$} & \multicolumn{3}{|c|}{$\begin{array}{l}D_{\mathrm{rc}} \\
300 \mathrm{~nm}\end{array}$} & \multicolumn{3}{|c|}{$\begin{array}{l}D_{\text {вас }} \\
400 \mathrm{~nm}\end{array}$} & \multicolumn{3}{|c|}{$\begin{array}{l}D_{\text {вас }} \\
500 \mathrm{~nm}\end{array}$} \\
\hline & $\begin{array}{l}\mathrm{D}_{\mathrm{D}} \\
5 \mu \mathrm{m}\end{array}$ & $\begin{array}{l}\mathrm{D}_{\mathrm{D}} \\
8 \mu \mathrm{m}\end{array}$ & $\begin{array}{l}\mathrm{D}_{\mathrm{D}} \\
12 \mu \mathrm{m}\end{array}$ & $\begin{array}{l}\mathrm{D}_{\mathrm{D}} \\
5 \mu \mathrm{m}\end{array}$ & $\begin{array}{l}\mathrm{D}_{\mathrm{D}} \\
8 \mu \mathrm{m}\end{array}$ & $\begin{array}{l}\mathrm{D}_{\mathrm{v}} \\
12 \mu \mathrm{m}\end{array}$ & $\begin{array}{l}\mathrm{D}_{\mathrm{b}} \\
5 \mu \mathrm{m}\end{array}$ & $\begin{array}{l}\mathrm{D}_{\mathrm{b}} \\
8 \mu \mathrm{m}\end{array}$ & $\begin{array}{l}\mathrm{D}_{\mathrm{D}} \\
12 \mu \mathrm{m}\end{array}$ & $\begin{array}{l}\mathrm{D}_{\mathrm{b}} \\
5 \mu \mathrm{m}\end{array}$ & $\begin{array}{l}\mathrm{D}_{\mathrm{b}} \\
8 \mu \mathrm{m}\end{array}$ & $\begin{array}{l}\mathrm{D}_{\mathrm{D}} \\
12 \mu \mathrm{m}\end{array}$ & $\begin{array}{l}\mathrm{D}_{\circ} \\
5 \mu \mathrm{m}\end{array}$ & $\begin{array}{l}\mathrm{D}_{\mathrm{b}} \\
8 \mu \mathrm{m}\end{array}$ & $\begin{array}{l}\mathrm{D}_{\mathrm{b}} \\
12 \mu \mathrm{m}\end{array}$ \\
\hline 0 & 0.00 & 0.00 & 0.00 & 0.00 & 0.00 & 0.00 & 0.00 & 0.00 & 0.00 & 0.00 & 0.00 & 0.00 & 0.00 & 0.00 & 0.00 \\
\hline 50 & 1.81 & 7.43 & 25.1 & 0.23 & 0.93 & 3.14 & 0.07 & 0.27 & 0.92 & 0.03 & 0.12 & 0.39 & 0.01 & 0.06 & 0.20 \\
\hline 100 & 3.63 & 14.9 & 50.1 & 0.45 & 1.86 & 6.28 & 0.13 & 0.55 & 1.85 & 0.06 & 0.23 & 0.79 & 0.03 & 0.12 & 0.40 \\
\hline 200 & 7.26 & 29.7 & 100 & 0.91 & 3.72 & 12.6 & 0.27 & 1.09 & 3.69 & 0.11 & 0.47 & 1.57 & 0.06 & 0.24 & 0.81 \\
\hline 400 & 14.5 & 59.4 & 201 & 1.82 & 7.45 & 25.1 & 0.53 & 2.19 & 7.38 & 0.23 & 0.93 & 3.15 & 0.12 & 0.48 & 1.62 \\
\hline 600 & 21.8 & 89.2 & 301 & 2.73 & 11.2 & 37.7 & 0.80 & 3.28 & 11.1 & 0.34 & 1.40 & 4.72 & 0.18 & 0.72 & 2.42 \\
\hline 800 & 29.0 & 119 & 401 & 3.64 & 14.9 & 50.3 & 1.07 & 4.38 & 14.8 & 0.46 & 1.87 & 6.29 & 0.23 & 0.96 & 3.23 \\
\hline
\end{tabular}

